\title{
Is Intraperitoneal Chemotherapy a New Strategy for Diffuse Abdominopelvic Tumor in Pediatric Patients?
}

\author{
Babak Abdolkarimi ${ }^{* 1}$, Dr. Mahdi Shahriari², and Dr. Soheila Zareifar ${ }^{3}$ \\ ${ }^{I}$ Assistant professor of Pediatric Hematology-Oncology, Lorestan University of Medical Sciences, Khoramabad, Iran \\ ${ }_{2}^{2}$ Associate Professor of Pediatric Hematology-Oncology, Department of Pediatrics, Shiraz University of Medical Sciences, Shiraz, Iran \\ ${ }^{3}$ Associate Professor of Pediatric Hematology-Oncology, Department of Pediatrics, Shiraz University of Medical Sciences, Shiraz, Iran
}

"Corresponding author: Babak Abdolkarimi, Assistant professor of Pediatric Hematology-Oncology, Lorestan University of Medical Sciences, Khoramabad, Iran, Tel: +989183605274;

E-mail: b.abdolkarimi@yahoo.com

Received Date: $21^{\text {st }}$ October 2017

Accepted Date: $19^{\text {th }}$ March 2018

Published Date: $25^{\text {th }}$ March 2018

\section{Dear editor}

Radiation therapy (RT) to abdominopelvic for tumors involved these sites may cause adverse effects such as rectal bleeding, incontinence, bladder irritation, and sexual problems in both men and women. Also probabale long-term effects include: Growth retardation and shortening of bones or a deformity in spine occur due to radiation.

In female population radiation may disturb the ovarian activity. This might lead to abnormal menstrual bleeding or problems in pregnancy ability. Radiation therapy also increases the risk of developing second malignancy in the some areas, many years later radiation exposure [1].

These harmful effects were persuaded the pediatric oncologist to find an alternative option against some childhood malignancies with peritoneal metastasis. Intraperitoneal chemotherapy may be was one of adjuvant or main strategy, against tumors involve abdominal cavity. Adult oncologists usually prescribe intraperitoneal chemotherapy (HIPEC) for the handling of ovarian cancer, but in pediatric oncology especially in Iran there isn't sufficient trial and experience.

MD Anderson was the first oncology center in the United States to use HIPEC on cancerous children with desmoplastic small round cell tumor (DSRCT) with diffuse peritoneal metastasis [2].

In DSRCT consolidative whole-abdomen radiotherapy at a dose of 30Gy, with or without a focal boost, is initially utilized after debulking surgery. However, significant hematological and gastrointestinal toxicities are observed. The intensity-modulated radiation therapy (IMRT) has resulted in a significant reduction in radiotherapy-related morbidity specifically
Citation: Abdolkarimi B,Shahriari,Zareifar S(2018) Is Intraperitoneal Chemotherapy a New Strategy for Diffuse Abdominopelvic Tumor in Pediatric Patients? Enliven: Challenges Cancer Detect Ther 5(1): 001.

Copyright: @ 2018 Babak Abdolkarimi. This is an Open Access article published and distributed under the terms of the Creative Commons Attribution License that permits unrestricted use, distribution and reproduction in any medium, provided the original author and source are credited.

gastrointestinal and hematologic toxicity. However, two groups at MSKCC (IMRT after debulking surgery) and MD Anderson (IMRT after debulking surgery and HIPEC) have reported sub-optimal disease-free survival of $<1$ year. Most relapses were intraperitoneal, suggesting that intraperitoneal therapy in addition to conventional surgery, chemotherapy and radiotherapy is necessary to improve outcome [3]

Hayes-Jordan A in 2012 evaluated toxicity of HIPEC with Cisplatin in 23 children Sincluded rhabdomyosarcoma (RMS), non-RMS soft tissue sarcoma, (NRSTS), desmoplastic small round cell tumor, (DSRCT), mesothelioma, Wilms tumor, melanomatosis, and adenocarcinoma. They were enrolled on dose escalation cohort received $150 \mathrm{mg} / \mathrm{m} 2$ of cisplatin. In this study 7 patients $(26 \%)$ had no recurrence [4].

A Hayes-Jordan used complete cytoreduction with HIPEC using $100 \mathrm{mg} /$ M2 of Cisplatin for 90 minutes in a closed technique and neoadjuvant chemotherapy. Of 101 pediatric CRS and HIPEC operations, 8 had ovarian primary tumors and multifocal peritoneal disease. The remaining $63 \%$ are disease free 2 to 6 years post HIPEC. Overall survival and relapse free survival in this cohort was $64 \%$ and $62 \%$ respectively [5].

Scalabre A in 2017 studies 22 patients with peritoneal mesotheliomas; seven, desmoplastic small round cells tumors (DSRCT); and other histologic types. In This study the mean overall survival (OS) and disease-free survival (DFS) were 57.5 months $(95 \% \mathrm{CI})$ and 30.9 months $(95 \% \mathrm{CI})$. Patients with a peritoneal mesothelioma had the best OS $(\mathrm{p}=0.015)$ and DFS $(\mathrm{p}=0.028)$ than other histologic type. 
Therefore base of this study HIPEC are encouraging for the treatment of peritoneal mesothelioma in children [6].

\section{How Intraperitoneal Chemotherapy is Done?}

Intraperitoneal chemotherapy or in distinct term Hyperthermic intraperitoneal chemotherapy (HIPEC) is done after Peritoneal dialysis catheter placement into the abdomen surgically.

The oncologists administer chemotherapy medications (Cisplatin and Paclitaxel main drugs are used for intraperitoneal chemotherapy) through this port lasts between two and three hours for approximately five to six months.

As shown in figure 1 , in the operating room, heated intraoperative intraperitoneal chemotherapy is used $\left(103^{\circ} \mathrm{F}\right.$ or $\left.42^{\circ} \mathrm{C}\right)$. Heat is part of the optimizing process and is administered to bring as much dose intensity to the abdominal and pelvic surfaces as is possible.

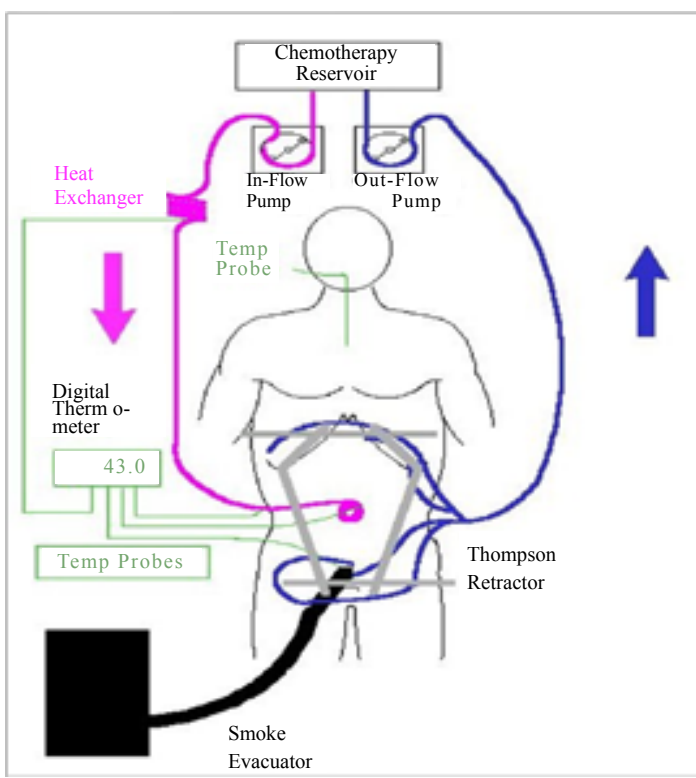

Figure1: Flow of HIPEC Treatment

Hyperthermia with intraperitoneal chemotherapy has several benefits: Such as more toxicity for malignant tissue than for normal tissue and better penetration of chemotherapy drugs into tissues and more cytotoxicity of selected chemotherapy agents [7].

This synergism occurs only at the accolade of heat and body tissue at the peritoneal surface.

Side effects of intraperitoneal chemotherapy include allergic reactions, nausea, vomiting, abdominal pain or bloating, shortness of breath, and decreased quantities of red or white blood cell.

HIPEC is performed after the surgeon resects tumor bulk or large lesions from mesentery, peritoneum pelvis or other parts of the abdominal area as it is possible; then HIPEC irradicates residual tumor and micro metastses [8].
In summary, HIPEC is a unique treatment done in the operating room, when standard chemotherapy and radiation therapy has limitation in adult and pediatric patients for soft tissue sarcomas, appendix cancer, Wilms' tumor and other cancers in the abdominal cavity. Although this method still needs more experience in children especially in IRAN that localized radiation therapy method isn't developed.

We suggest this method as an alternative method for all of abdominal solid tumors which complete radiation therapy compliance.

\section{References}

1. Ramuz O, Bourhis J, Mornex F (1997) Late effects of radiations on mature and growing bone, Cancer Radiother 1: 801-809.

2. Ramarajapalli ML, Kilara NG, Subramanyam M, Rao NA (2011) Technique of intraperitoneal chemotherapy using veress needle in patients with ovarian cancer. Int J Gynecol Cancer 21: 1388-1390.

3. Hayes-Jordan A, Lopez C, Green HL, Xiao LC, Huh W, et al. (2016) Cytoreductive Surgery (CRS) and Hyperthermic Intraperitoneal Chemotherapy (HIPEC) in Pediatric Ovarian Tumors: A Novel Treatment Approach. Pediatr Surg Int 32: 71-73.

4. Hayes-Jordan A, Green H, Ludwig J, Anderson P (2012) Toxicity of hyperthermic intraperitoneal chemotherapy (HIPEC) in pediatric patients with sarcomatosis/carcinomatosis: early experience and phase 1 results. Pediatr Blood Cancer 59: 395-397.

5. Honore C, Atallah V, Mir O, Orbach D, Ferron G, et al. (2017) Abdominal desmoplastic small round cell tumor without extraperitoneal metastases: Is there a benefit for HIPEC after macroscopically complete cytoreductive surgery? PLoS One 12: e0171639.

6. Scalabre A, Philippe-Chomette P, Passot G, Orbach D, Elias D, et al. (2018) Cytoreductive surgery and hyperthermic intraperitoneal perfusion with chemotherapy in children with peritoneal tumor spread: A French nationwide study over 14 years. Pediatr Blood Cancer 65.

7. Wydra D, Sawicki S, Ciach K, Marciniak A, Emerich J (2007) Combination of intraperitoneal hyperthermic perfusion chemotherapy (IHPC) with intraperitoneal chemotherapy as a treatment modality for persistent ovarian cancer. Eur J Gynaecol Oncol 28: 128-130.

8. Aarts F, Bleichrodt RP, de Man B, Lomme R, Boerman OC (2008) The effects of adjuvant experimental radioimmunotherapy and hyperthermic intraperitoneal chemotherapy on intestinal and abdominal healing after cytoreductive surgery for peritoneal carcinomatosis in the rat. Annals of surgical oncology 15: 3299-3307.

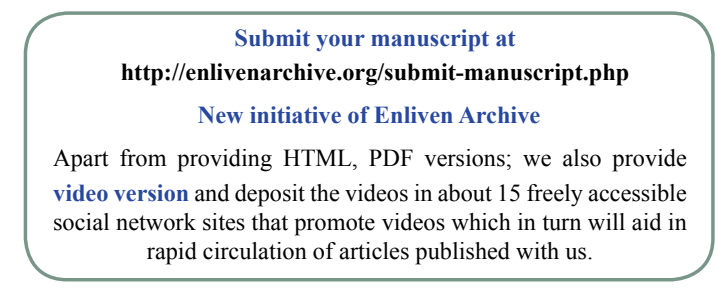

